\title{
Impact du système du contrôle interne sur la performance de la fonction commerciale : Cas des entreprises marocaines
}

\author{
Abdelali BABAALI', Fatima Zahra ACHOUR ${ }^{2}$ \\ ${ }^{1}$ Université Ibn Tofaïl, Kenitra, Maroc \\ ${ }^{2}$ Université Ibn Tofaïl, Kenitra, Maroc
}

\begin{abstract}
Résumé : Les chefs d'entreprises marocaines sont de plus en plus préoccupées par le phénomène de baisse d'activité commerciale, confirmé par les baromètres de conjoncture publiées par la CGEM durant les quatre dernières années (2015-2018), et dont les causes directes peuvent être liées à la concurrence déloyale, au problème des délais de paiements, aux créances impayées et enfin à l'existence d'un secteur informel pesant. A traves cet article, nous allons essayer de mettre l'accent sur la relation pouvant exister entre les éléments constitutifs d'un système de contrôle interne mis en place dans une fonction commerciale et sa performance. Autrement dit, nous allons tenter d'expliquer la non performance de la fonction commerciale par le recours à l'évaluation du système de contrôle interne qui y est déployé. Pour ce faire, nous allons présenter dans la première partie, les fondements théoriques des concepts 'système de contrôle interne et ' performance socio-économique. Ensuite, nous présentons les points de liaison et d'influence pour enfin élaborer des hypothèses qui feront l'objet d'une étude empirique qui portera sur un échantillon constitué de cent soixante et un entreprises marocaines. Les résultats de notre travail vont permettre d'apporter une réponse sur le degré d'influence de chaque dispositif faisant partie du système de contrôle interne de la fonction commerciale sur sa performance socio-économique et plus précisément sur sa performance commerciale. L'apport effectif de cette contribution se résume dans la confirmation de l'influence positive et significative de chaque dispositif constitutif d'un système de contrôle interne d'une fonction commerciale, en l'occurrence les objectifs, les moyens, le système d'information, l'organisation, les procédures et la vérification, et ce à travers l'étude menée auprès de 161 entreprises marocaines.
\end{abstract}

Mots-clés : Système de contrôle interne, performance socio-économique, performance commerciale Référentiel COSO.

\section{Introduction}

Dans un contexte d'ouverture économique, et sur la base des études menées par la CGEM [1] et plus précisément dans ses rapports publiés sous le nom ' baromètre de conjoncture' un nombre important des chefs d'entreprises marocaines exprime, durant les quatre dernières années, une mauvaise perception due 
essentiellement à la non performance de leurs entreprises constatées notamment au niveau de leurs fonctions commerciales. Les causes avancées par les dirigeants entreprises interrogés lors des études menées se résument dans la baisse de l'activité économique, la concurrence déloyale, le problème de délais de paiements, les créances impayées et enfin l'existence d'un secteur informel pesant.

Le problème de la non performance des fonctions commerciales des entreprises marocaines, manifesté par les causes citées en ci-dessous, a suscité notre curiosité de chercheur et nous a conduit à aller chercher dans les raisons qui pourrait éventuellement expliquer la contribution d'un système de contrôle interne mis en place au sein d'une fonction commerciale dans l'amélioration de sa performance. Pour ce faire, nous allons nous nous poser préalablement les questions suivantes :

Les entreprises marocaines sont-elles préoccupées par le souci de contrôle des activités au niveau de leurs fonctions commerciales?

Les entreprises marocaines veillent-elles à ce que leurs fonctions commerciales soient dotées d'un système de contrôle internes qui leurs sont propres et qui participent à la maîtrise de l'ensemble des activités ?

A notre connaissance et sur la base des recherches effectuées, il n'existe aucun travail scientifique expliquant la performance de la fonction commerciale des entreprises marocaines par l'efficacité du système de contrôle interne qui y est instauré.

Selon une approche institutionnaliste, et dans une économie ouverte en pleine mutation, les mauvaises performances de la fonction commerciale au sein des entreprises marocaines pourraient être alors expliquées par une absence d'un système de contrôle interne efficace ou l'existence d'un système de contrôle interne non pertinent

Le présent article vise à répondre à la problématique suivante :

Dans quelle mesure le système de contrôle interne influence les performances de la fonction commerciale au sein des entreprises ayant enregistré une baisse de l'activité et agissant dans un contexte de concurrence déloyale, de problème de délais de paiement et des créances impayées et d'un secteur informel pesant ?

Sur la base de notre problématique de recherche et dans les conditions économiques citées ci-dessus, notre objectif principal, par le biais de cette contribution, est de comprendre l'impact de la mise en place d'un système de contrôle interne au niveau de la fonction commerciale sur sa performance.

Pour ce faire, nous essayerons d'organiser le travail de la façon suivante :

Tout d'abord, dans une première partie, nous allons présenter les fondements de théorique du système de contrôle interne et celui de la performance organisationnelle en se référant aux ouvrages pour donner des définitions générales.

Aussi, nous essayerons de mobiliser une théorie pour élaborer une définition conceptuelle qui nous aidera à extraire facilement les hypothèses pour enfin conclure avec un modèle théorique de ce travail de recherche.

Ensuite, dans une deuxième partie, nous essayerons de cerner les différentes variables d'un système de contrôle interne, et ce par un recours aux travaux empiriques des recherches antérieures pour concevoir enfin notre modèle conceptuel. 
Après, dans une troisième partie, nous allons examiner l'influence des différentes composantes d'un système de contrôle interne d'une fonction commerciale par une étude quantitative portant sur un échantillon de 161 entreprises marocaines.

Enfin, nous présenterons les résultats obtenus avec une discussion détaillée de la réponse apportée à notre problématique.

\section{Le système de contrôle interne : historique des principales définitions et nouveau rôle}

Plusieurs définitions, élaborées par différents organismes et comités, ont été relatées dans la majorité des travaux de recherche scientifique portant sur thématique du contrôle interne. Dans le présent travail, nous avons essayé de sélectionner les définitions les plus répondues et nous les avons classées selon un ordre chronologique pour mettre en exergue un historique qui trace l'évolution de ce concept depuis son apparition.

La première définition du contrôle interne ayant connu un véritable succès du fait qu'elle soulignait un réel dépassement du cadre comptable habituel qui se basait uniquement sur la vérification financière est l'œuvre de l'Ordre des experts-comptables français en 1977. Ce dernier a partagé une vision plus large du contrôle interne en le définissant comme suit «Le contrôle interne est l'ensemble des sécurités contribuant à la maîtrise de l'entreprise. Il a pour but d'un côté d'assurer la protection, la sauvegarde du patrimoine et la qualité de l'information, de l'autre d'appliquer les instructions de la direction et de favoriser l'amélioration des performances. Il se manifeste par l'organisation, les méthodes et les procédures de chacune des activités de l'entreprise, pour maintenir la pérennité de celle-ci. » (Renard,2012) [2]

La deuxième célèbre définition, date de 1992, est établie par The Committee Of Sponsoring Organizations of the Treadway Commission. En fait, il s'agit d'une commission à but non lucratif ayant établit une définition standard du contrôle interne et a créé un cadre pour évaluer son efficacité. Par extension ce standard s'appelle aussi COSO. Pour cette commission « le contrôle interne est un processus mis en œuvre par le conseil d'administration, les dirigeants et le personnel d'une organisation destinée à fournir une assurance raisonnable quant à la réalisation des objectifs ».

En fait, l'apport particulier de cette définition est qu'elle implique toutes les ressources de l'entreprise depuis la base de l'organisation à son sommet. Chaque collaborateur, depuis son niveau hiérarchique, à son contrôle interne à surveiller et respecter pour converger à la maitrise des activités et la réalisation des objectifs fixés.

La troisième définition a été conçue en 1995 par le Canadian Institute of chartered accountants. Cet institut décrit le contrôle comme étant "Éléments de l'organisation (incluant ressources, systèmes, procédés, culture et tâches) qui, mis ensemble, aident à atteindre les objectifs ». A souligner que La particularité de cette définition est qu'elle a mis l'accent sur le volet moyen et par conséquent elle vient compléter celle du COSO.

En plus des trois définitions citées en ci-dessus, nous ajoutons la définition reportée dans le guide de contrôle interne (rapport publié en 1999), connu sous le nom «the Turnbull guidance » qui reprend l'essentiel des définitions COSO et COCO en les complétant par des objectifs. Selon ce guide, le contrôle interne comme étant un système qui englobe les politiques, processus, tâches, comportements et autres aspects qui sont combinés ensemble en vue de :

- Faciliter l'efficacité et l'efficience des opérations ; 
- Aider à assurer la qualité du reporting interne et externe ;

- Et aider à assurer la conformité aux lois et réglementations.

En 2006, l'autorité des marchés françaises a défit le contrôle interne via un usage des définitions de base comme " un dispositif de la société, défini et mis en œuvre sous sa responsabilité. Il comprend un ensemble de moyens, de comportements, de procédures et d'actions adaptés aux caractéristiques propres de chaque société qui

Contribue à la maîtrise de ses activités, à l'efficacité de ses opérations et à l'utilisation efficiente de ses ressources ;

Doit lui permettre de prendre en compte de manière appropriée les risques significatifs, qu'ils soient opérationnels, financiers ou de conformité. » (Renard, 2012, P5). [2]

Après avoir passé en revue les principales définitions du contrôle interne, il nous semble important de s'orienter vers le détail des dispositifs qui constituent les piliers d'un système de contrôle interne. Pour ce faire, nous allons faire recours aux travaux réalisés par (Renard, 2012) pour essayer de voir l'impact de chaque dispositif (y compris les facteurs d'influence) sur la performance de la fonction commerciale des entreprises, et ce pour pouvoir répondre à notre problématique. Selon Renard, 2012. P128), [2] un système de contrôle interne se regroupe dans six famille de dispositifs à savoir :

- Les objectifs ;

- Les moyens ;

- Les systèmes d'information ;

- L'organisation ;

- Les procédures ;

- La Vérification.

\section{Performance socio-économique et performance commerciale}

(Salgado, 2013) a essayé de mettre sous la lumière le concept de la performance et a conclu qu'il s'agit d'un mot « valise » dans le sens où toutes ses définitions restent floues et multidimensionnelles. [3]

Dans le présent travail, nous allons nous limiter à la revue de littérature relative à une seule dimension de la performance. A savoir la performance socio-économique. Cette dernière englobe à son tour quatre catégories qui sont la performance organisationnelle, la performance sociale, la performance économique et financière et enfin la performance commerciale que nous cherchons à expliquer via les familles de dispositifs cités ci-dessus.

Premièrement, la performance organisationnelle nous informe sur la façon par laquelle une entreprise est organisée pour réaliser ses objectifs. (Bocco, 2010). [4]

Deuxièmement, la performance sociale, comme son nom l'indique, informe sur la nature des liens et relations sociaux au sein de l'entreprise et renseigne sur l'intérêt que porte l'entreprise au volet social. Dans ce sens, la performance sociale peut être mesurée par la qualité des liens sociaux entre les entités, les responsables et les employés. (Marmuse, 1997, cité Bocco, 2010). [4]

Troisièmement, la performance économique appelée aussi financière reste, pour un nombre important de dirigeants, le seul critère d'évaluation et de performance de leurs entreprises. En effet, les expériences ont démontré que pas mal de cas d'échec de certaines entreprises ne sont pas dus essentiellement à une non performance financière et que leurs maitrises des activités étaient derrière leurs défaillances 
entrainant ainsi une baisse du chiffres d'affaires réalisé et par conséquent une non performance commerciale.

Dans ce sillage, le concept de la performance commerciale a pris une petite place dans les travaux de chercheurs et la majorité le traite dans son sens global. Dans ce travail, nous avons essayé de faire un focus sur la littérature ayant porté sur ce type particulier de performance tels que (Furrer \& Sudharshan, 2003, Cité par Bocco2010) [4] et (Bughin, 2006, Cité par Bocco2010) [4]. En fait, les auteurs de ces travaux lient la performance commerciale d'une manière générale à la satisfaction des clients finaux.

Aussi, (Croteau et al., 2001) [5], a souligné que la performance d'une fonction commerciale peut être mesurée en se référant à trois dimensions. A savoir :

L'efficacité (comparaison entre les objectifs commerciaux et les résultats réalisés);

L'efficience (comparaison entre les moyens mobilisés et les résultats de la fonction);

La pertinence (la comparaison entre les moyens mobilisés et les objectifs de la fonction).

\section{Dispositifs du contrôle interne et performance commerciale : quelle relation ?}

Selon (Renard,2012), un système de contrôle interne regroupe six familles de dispositifs [2]. Dans un premier temps, nous aborderons la famille Objectifs. En effet, la fixation des objectifs représente la première référence à laquelle chaque responsable réserve un intérêt particulier. Prenons l'exemple de la fonction commerciale, une équipe appelée à réaliser des ventes doit se référer à des seuils minimums que ce soient quantitatifs ou qualitatifs qui permettent de mesurer la productivité de l'équipe et d'avoir une idée sur le niveau d'engagement et le degré d'implication de chaque collaborateur pour pouvoir les départager lors des évaluations. Nous pouvons avancer à ce stade que les objectifs donnent lieu à la compétition et par conséquent à un état d'esprit d'excellence qui entraînera sans doute une certaine performance.

Selon la thèse de March (1978), les limitations des individus travaillant à la fonction commerciale seraient des limites de la capacité de calcul des conséquences futures de leurs actions actuelles, vue les complications de la prévision des conséquences futures, et les décisions prévisionnelles liées à ces conséquences, de l'organisation de ces décisions et de l'utilisation de leurs mémoires. March (1978) suggère alors le développent des procédures de décision qui sont raisonnables et qui prennent en compte les contraintes de la fonction. Sur cette base, nous élaborons l'hypothèse suivante :

\subsection{Hypothèse-1 : l'absence des objectifs pourrait avoir un impact négatif sur la performance de la fonction commerciale.}

(Renard,2012) souligne également que le dispositif de contrôle 'Objectifs', mis en place au sein de la fonction commerciale, ne servira à rien dans les cas où :

- Les objectifs de la fonction commerciale ne sont pas connus et communiqués ;

- Les risques pouvant nuire aux objectifs de la fonction commerciale ne sont pas identifiés ;

- Non fixation d'une politique au sein de la fonction commerciale pouvant réaliser les objectifs et gérer les risques ;

- Des plans d'action pouvant réaliser cette politique ne sont pas établis.

Sur cette base, nous allons extraire les quatre sous-hypothèses liée à notre première hypothèse citée en ci-dessus : 
Sous hypothèse-1.1 : L'ignorance et la non communication des objectifs fixés aux fonctions commerciales influenceraient négativement la performance de ces dernières.

Sous hypothèse-1.2 : La non identification des risques inhérents aux activités des fonctions commerciales pourrait avoir un impact négatif sur la performance de ces dernières.

Sous hypothèse-1.3 : L'absence de politique pour réaliser les objectifs des fonctions commerciales influencerait la performance de ces dernières

Sous hypothèse-1.4 : L'absence des plans d'action pour déployer les politiques des fonctions commerciales influencerait négativement la performance de ces dernières.

La deuxième famille des dispositifs est les Moyens. Il y a lieu de souligner que la mise en place du dispositif de contrôle 'objectifs' est derrière la fixation des moyens. Dans ce sens, plusieurs constats d'anomalies émis par les auditeurs internes sont dus essentiellement à une insuffisance ou à une inadaptation des moyens mis à disposition pour atteindre les objectifs fixés.

Sur cette base, nous pouvons élaborer notre deuxième hypothèse :

\subsection{Hypothèse-2 : l'absence des moyens pourrait avoir un impact négatif sur la performance de la fonction commerciale.}

Aussi, le concept Moyens englobe plusieurs formes et catégories. Dans les travaux de recherche antérieurs, notamment ceux empiriques, parler des moyens renvoie souvent à une séparation par type de ces derniers. Nous citons par exemple les moyens humains, les moyens financiers, les moyens techniques et les moyens commerciaux.

Dans ce sens, nous pouvons formuler les sous-hypothèses ci-dessous :

Sous hypothèse 2.1: L'absence de moyens humains pour atteindre les objectifs fixés aux fonctions commerciales influencerait négativement la performance de ces dernières.

Sous hypothèse 2.2: L'absence de moyens financiers pour atteindre les objectifs fixés aux fonctions commerciales influencerait négativement la performance de ces dernières.

Sous hypothèse 2.3: L'absence de moyens techniques pour atteindre les objectifs fixés aux fonctions commerciales influencerait négativement la performance de ces dernières.

Sous hypothèse 2.4: L'absence de moyens commerciaux pour atteindre les objectifs fixés aux fonctions commerciales influencerait négativement la performance de ces dernières.

Dans un troisième temps, nous aborderons la famille de dispositifs de contrôle interne la plus sensible et la plus importante notamment dans les structures complexes. Il s'agit là des Systèmes d'information. L'information et la communication occupent un rôle vital pour la réussite de toute coordination et sont la base essentielle de toute prise de décision. Les systèmes d'information fournissent aux acteurs du contrôle interne l'information qui fera l'objet d'une évaluation et par conséquent à une mobilisation. Pour les autres, ils sont dispensables dans le sens où toutes les activités de l'entreprise sont coordonnées et suivies via l'information et sa communication. En effet, sans ces dernières, le système de contrôle interne sera voué à l'échec, dans la mesure où toutes les personnes chargées de sa mise en œuvre n'en seront pas informées OUTSEKI J. (2019). Aussi, la décision basée sur les informations extraites des systèmes d'information ne pourra pas être prise en l'absence desdits systèmes d'information (Rittenberg, Larry E, 2006). [6] 
Dans la pratique, nous ne pouvons pas parler de l'efficacité d'un système d'information considéré en tant que dispositif de contrôle interne au sein d'une fonction commerciale que s'il est capable de remplir les conditions suivantes :

- Des tableaux de bord de suivi d'activité de la fonction commerciale sont installés.

- Des tableaux de bord de gestion de la fonction commerciale sont installés.

- Des tableaux de bord de pilotage commerciale sont installés.

En dépit de ça, nous pouvons dire que l'efficacité des systèmes d'information pourrait garantir une disponibilité permanente des information fiables et utiles qui serviront de canaux de données qui permettront aux responsables opérationnels de maîtriser davantage l'ensemble des activités et de garantir la performance recherchée.

Sur cette base, notre troisième hypothèse sera :

\subsection{Hypothèse-3 : l'absence des systèmes d'information pourrait avoir un impact négatif sur la performance de la fonction commerciale.}

Comme présenté ci-dessus, les variables pouvant impacter positivement ou négativement l'apport des systèmes d'information en tant que dispositif de contrôle interne sont : les tableaux de bord de suivi, de gestion et enfin de pilotage. De plus, le pilotage est le sommet du modèle COSO (2013). Il suppose que l'efficacité du processus de contrôle interne est liée à son évaluation périodique et/ou ponctuelle et de communiquer les faiblesses en interne de l'organisation afin de mettre en place des actions correctives (boucle de retour) (Noiret et Walter, 2008 ; Vaassen et al, 2009 ; Ki-zerbo, 2013, cité par OUASHIL \& OUHADI, 2019). [7]

C'est ce qui nous amène à élaborer les trois sous--hypothèses ci-dessous :

Sous hypothèse 3.1: L'absence des tableaux de bord de suivi des activités des fonctions commerciales influencerait négativement la performance de ces dernières.

Sous hypothèse 3.2: L'absence des tableaux de bord de gestion des activités des fonctions commerciales influencerait négativement la performance de ces dernières.

Sous hypothèse 3.3: L'absence des tableaux de bord de pilotage des activités des fonctions commerciales influencerait négativement la performance de ces dernières.

La quatrième famille de dispositifs de contrôle interne est l'Organisation. Fayolle disait que nous ne pouvons contrôler que ce qui est organisé (Renard, 2012, P.135) [2]. A partir de cette citation, nous pouvons conclure que le contrôle est étroitement lié à l'organisation. Aussi, selon le même auteur, une bonne organisation est tributaire de trois variables, à savoir :

- Organisation adaptée et logique : pour qu'une fonction commerciale serait performante, son organisation devrait être adaptée aux types d'activité, à l'environnement et surtout à la culture.

- Organisation objective et raisonnée : pour qu'une fonction commerciale serait performante, son organisation devrait tenir compte des compétences de ses ressources et les faire placer des dans postes cohérents avec leurs formations de base.

Organisation basée sur la répartition des tâches : Si un responsable commercial compte attribuer à la même personne des tâches qui sont incompatibles. Ce dernier court le risque de ne pas contrôler 
l'ensemble de l'activité et par conséquent donner lieu à des situations de fraude. Le contrôle est une culture basée d'abord sur la séparation des tâches en général et celles incompatibles en particulier.

Ceci dit, l'organisation adoptée au sein des fonctions commerciales présenterait le critère essentiel pour espérer à une performance de ces dernières. Dans ce sens, nous élaborons notre quatrième hypothèse et les trois sous-hypothèses qui en ressortent :

\subsection{Hypothèse-4 : l'absence de l'organisation pourrait avoir un impact négatif sur la performance de la fonction commerciale.}

Sous hypothèse 4.1: L'absence d'organisation adaptée au sein des fonctions commerciales influencerait négativement la performance de ces dernières.

Sous hypothèse 4.2: L'absence d'organisation objective au sein des fonctions commerciales influencerait négativement la performance de ces dernières.

Sous hypothèse 4.3: L'absence d'organisation basée sur la séparation des tâches au sein des fonctions commerciales influencerait négativement la performance de ces dernières. La cinquième famille de dispositifs est les Procédures. En fait, toute activité de contrôle englobe des normes et des procédures déployées au sein de l'entreprise pour anticiper sur les risques susceptibles d'entraver l'atteinte des objectifs fixés (Mandzila, 2004) [8]. Une absence des procédures qui tracent l'ensemble des contrôles à faire par les opérationnels au sein de toutes les activités liées à la fonction commerciale entrânerait une non atteinte des objectifs et par conséquent une non performance de la fonction toute entière d'où l'hypothèse suivante :

\subsection{Hypothèse 5 : l'absence des procédures pourrait avoir un impact négatif sur la performance de la fonction commerciale.}

Quant à la sixième et dernière famille de dispositifs, en l'occurrence la Vérification. Ce dispositif renvoie sur la supervision exercée la hiérarchie directe qui est appelée à s'assurer du niveau de maîtrise des activités de l'entreprise. Il s'agit là d'un contrôle du premier niveau par lequel les responsables opérationnels démontrent à leurs équipes que leurs actes sont vérifiés et suivis. Cet exercice réalisé selon une fréquence aléatoire favorise le respect des procédures de fonctionnement, permet d'éviter la survenance des risques identifiés et contribuerait à la performance des fonctions de l'entreprise y compris celle commerciale. Cette liaison nous permettra d'élaborer notre cinquième hypothèse :

\subsection{Hypothèse 5 : l'absence de vérification pourrait avoir un impact négatif sur la performance de la fonction commerciale.}

Aussi, il est important de citer les facteurs susceptibles créer une négligence de la part des responsables de vérification tels que la confiance démesurée dans les équipes, l'absence de matérialisation des vérifications effectuées, l'incompétences et l'habileté des acteurs chargés de la vérification et enfin le mode de supervision (sur place ou à distance).

L'ensemble de ces facteurs donnera lieu aux sous-hypothèses ci-dessous :

Sous hypothèse 5.1: L'absence de la supervision suite à la confiance en les équipes des fonctions commerciales influencerait négativement la performance de ces dernières.

Sous hypothèse 5.2: L'absence de la matérialisation des vérifications réalisées au sein des fonctions commerciales influencerait négativement la performance de ces dernières. 
Sous hypothèse 5.3: L'absence de compétence et des habilités des vérificateurs des activités des fonctions commerciales influencerait négativement la performance de ces dernières.

Sous hypothèse 5.4: Le mode de vérification (sur place /à distance) des activités au niveau des fonctions commerciales influencerait négativement la performance de ces dernières.

Finalement, sur la base de notre revue de littérature, la non performance des fonctions commerciales serait le résultat de :

- L'ignorance et la non communication des objectifs fixés aux fonctions commerciales.

- L'absence des moyens humains, financiers, techniques et commerciaux nécessaires à la réalisation des objectifs fixés.

- L'absence d'un système d'information permettant de fournir des tableaux de bord de suivi, de gestion et de pilotage des activités de la fonction commerciale.

- L'absence de l'organisation adaptée et objective en évitant tout cumul des tâches incompatibles par la même ressource.

- Enfin, l'absence de vérification directe et indirecte.

Après avoir formulé les six hypothèses sur les relations entre les différentes variables de notre modèle, nous allons concevoir graphiquement notre modèle conceptuel d'analyse empirique des données portant sur la performance de la fonction commerciale pour essayer de le vérifier par la suite auprès de notre échantillon d'entreprises marocaines.

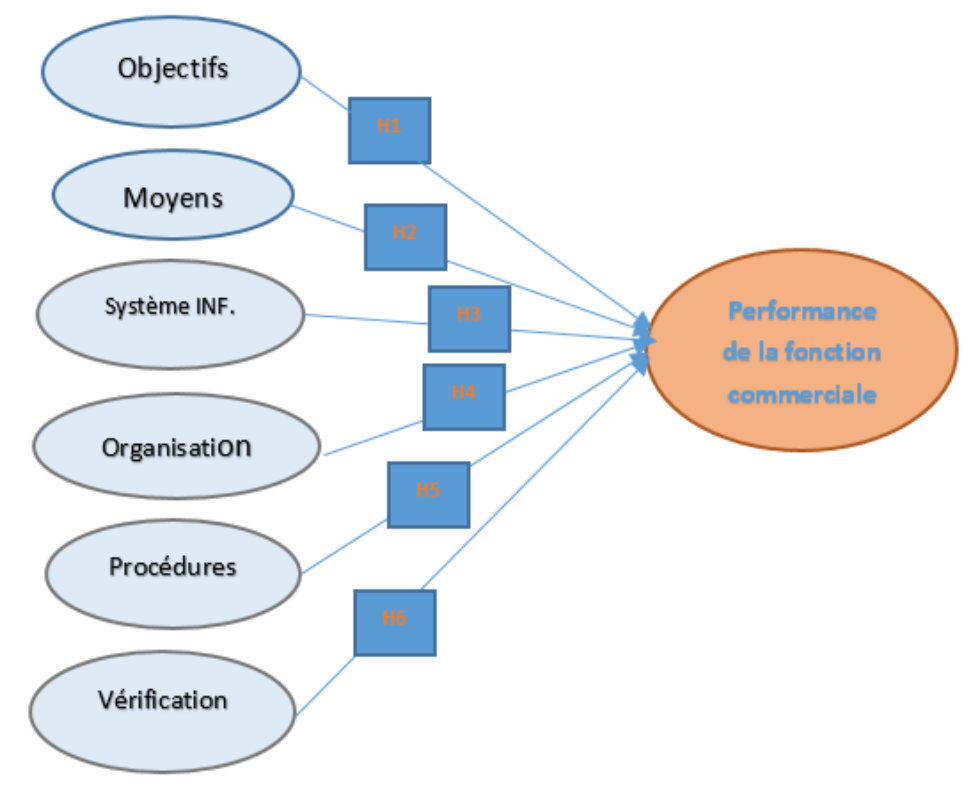

Figure 1 : Modèle conceptuel de recherche (MCR).

\section{Résultats de l'étude empirique}

L'étude empirique menée a porté sur un échantillon de 161 entreprises marocaines disposant toutes d'une structure d'audit interne et des départements commerciaux et nous y trouverons, également, des cabinets d'audit spécialisés ayant réalisés des missions d'audit interne et de conseil. Notre objectif, via cette étude quantitative, est la validation des hypothèses émises ci-dessus en faisant recours des tests statistiques et des échelles de mesure validées.

Rappelons notre première hypothèse de départ relative à L'ignorance et la non communication des objectifs fixés aux fonctions commerciales. Les résultats des statistiques uni variées ont démontré qu'un grand nombre d'entreprises de l'échantillon interviewé confirme que les objectifs sont fixés et déclinés à 
leurs fonctions commerciales, soit (91.8\% des réponses). Au sein des fonctions commerciales des entreprises marocaines, les objectifs sont fixés et déclinés par référence à des politiques qui garantissent leurs atteintes et à des analyses des risques qui peuvent entraver leurs réalisations. En dépit de ça, les objectifs en tant que dispositif de contrôle interne impactent positivement et significativement la performance de la fonction commerciale au sein des entreprises marocaines.

La deuxième hypothèse est relative à l'absence des moyens humains, financiers, techniques et commerciaux nécessaires à la réalisation des objectifs fixés. Les résultats statistiques ont fait ressortir que 89.13\% des responsables des départements commerciaux interrogés confirment la disponibilité des moyens humains, financiers, commerciaux et techniques nécessaires à la réalisation des opérations engagées au niveau de leurs différentes activités. Sur cette base, nous pouvons confirmer qu'au Maroc, la performance des fonctions commerciales est assurée par la disponibilité des moyens humains, financiers, commerciaux et techniques nécessaires à l'exécution des plans d'actions fixés.

La vérification de la troisième hypothèse relative à l'absence des systèmes d'information et son impact sur la performance de la fonction commerciale a fait ressortir ce qui suit, et ce à partir des réponses fournies :

- La majorité écrasante des entreprises marocaines de l'échantillon étudié dispose des systèmes d'information à travers lesquels ces derniers éditent des reporting journaliers de suivi de leurs activités.

- Au Maroc, les entreprises suivent les réalisations de leurs objectifs commerciaux à l'aide des tableaux de bord de gestion et la réalisation des plans d'actions engagés à l'aide des tableaux de bord de pilotage. Soit $95 \%$ de notre échantillon.

La quatrième hypothèse relative à l'absence de l'organisation comme dispositif de contrôle interne et son influence sur la performance de la fonction commerciale a été validée et les résultats obtenus le confirment. Ceci dit, selon les réponses obtenues, la majorité des responsables des départements commerciaux (91.6\%) sont d'accord qu'au sein de leurs fonctions commerciales existent une structure objective (organigramme hiérarchique) qui prend en considération le principe de la séparation des tâches.

Quant aux procédures et leur impact sur la performance de la fonction commerciale, l'étude menée a confirmé que les entreprises marocaines s'accordent sur l'évidence que leurs activités au sein de leurs fonctions commerciales sont régies par des procédures écrites qui servent à mieux maîtriser les opérations de gestion de chaque activité.

Finalement, l'impact du dispositif de vérification, instauré au sein des fonctions commerciales, sur la performance de ces dernières est justifiée dans le sens où une majorité représentée par un pourcentage de $89.01 \%$ des responsables opérationnels marocains confirment de la réalisation des opérations de contrôle assurées par les managers directs et indirects avec une traçabilité et une matérialisation de constats pour pallier à tout risque qui pourra donner lieu à une contre-performance.

\section{Conclusion}

Sur la base de notre étude empirique, la réponse à notre problématique posée dans le cadre de cette contribution scientifique est que le système de contrôle interne tel qu'il présenté par ses six familles de dispositif de contrôle, à savoir les objectifs fixés, les moyens, les systèmes d'information, l'organisation, les procédures et la vérification influence significativement et positivement la performance des fonctions commerciales. Ceci dit, nous avons pu confirmer que l'instauration de ces dispositifs garantira la maitrise 
des opérations au sein de chaque activité commerciale et par conséquent l'efficacité du système de contrôle interne et la performance de la fonction toute entière.

\section{BIBLIOGRAPHIE}

[1] Baromètres de conjoncture publiées par la CGEM https://www.cgem.ma

[2] Renard, J. (2012). Comprendre et mettre en œuvre le contrôle interne (1 éd., vol.1, p.13-25). Eyrolles Editions d' Organisation: Paris.

[3] Salgado, M. (2013). Analyse des fondamentaux de la performance et de sa valorisation.

[4] ] Bocco, B. S. (2010). Perception de la notion de performance par les dirigeants de petites entreprises en Afrique. La Revue des Sciences de Gestion, (1), 117-124.

[5] Croteau, A. M., RAYMOND, L \& Bergeron, F. (2001). Comportements stratégiques, choix et gestion des systèmes d'information : Contribution à la performance. Systèmes d'information et management, 6(4).

[6] OUTSEKI J. (2019) «L'influence des composantes du système de contrôle interne selon le cadre de référence COSO sur la performance de l'entreprise : une exploration théorique » Revue Internationale des Sciences de Gestion « Numéro 3 : Avril 2019 / Volume 2 : numéro 2 » : 635- 650.

[7] OUASHIL M. \& OUHADI S. (2019) « Le contrôle interne face à l'émergence de nouvelles formes des risques : cas de la fraude » Revue Internationale des Sciences de Gestion « Numéro 3 : Avril 2019 / Volume 2 : numéro 2 » p : 805 819.

[8] Mandzila, E. E. W. (2004). LA CONTRIBUTION DU CONTROLE INTERNE ET DE L'AUDIT AU GOUVERNEMENT D'ENTREPRISE (Doctoral dissertation, UNIVERSITE PARIS XII VAL de MARNE). 